\title{
THE PRESCRIBED $p$-MEAN CURVATURE EQUATION OF LOW REGULARITY IN THE HEISENBERG GROUP
}

\author{
JIH-HSIN CHENG
}

\begin{abstract}
I will report on recent study about regularity and the singular set of a $C^{1}$ smooth surface with prescribed $p($ or $H)$-mean curvature in the 3-dimensional Heisenberg group. As a differential equation, this is a degenerate hyperbolic and elliptic P.D.E. of second order, arising from the study of $C R$ geometry. Assuming only the $p$-mean curvature $H \in C^{0}$, we showed that any characteristic curve is $C^{2}$ smooth and its (line) curvature equals $-H$. By introducing special coordinates and invoking the jump formulas along characteristic curves, we proved that the Legendrian (horizontal) normal gains one more derivative. Therefore the seed curves are $C^{2}$ smooth. We also obtained the uniqueness of characteristic and seed curves passing through a common point under some mild conditions, respectively. In an on-going project, we showed that the $p$-area element is in fact $C^{2}$ smooth along any characteristic curve and satisfies a certain ordinary differential equation of second order. Moreover, we analyzed this O.D.E. to study the singular set.
\end{abstract}

\section{Regularity AND CURVATURE OF CHARACTERISTIC CURVES}

A pseudohermitian manifold $(M, J, \Theta)$ is an odd dimensional $\left(C^{\infty}\right.$ smooth $)$ manifold $M$ (say, of dimension $2 n+1$ ) together with a contact form $\Theta$, i.e. a 1-form satisfying $\Theta \wedge(d \Theta)^{n} \neq 0$ everywhere, and an almost complex structure $J$, i.e. an endomorphism from the contact bundle $\xi \equiv \operatorname{ker} \Theta$ onto itself satisfying $J^{2}=-I$ dentity (usually $J$ is also required to satisfy a certain integrability condition while, for $n=1$, this condition always holds) (see, e.g., [10]). The natural examples arise as the boundaries of strongly pseudoconvex domains in $C^{n+1}$. Let $D$ be a strongly pseudoconvex domain in $C^{n+1}$. Let $M=\partial D$. We take $\Theta=-i \partial \varphi(i=\sqrt{-1})$ restricted to $M$, where $\varphi$ is a defining function for $M$. It follows that the contact bundle $\xi$ coincides with $T M \cap J_{C^{n+1}} T M$ where $J_{C^{n+1}}$ denotes the almost complex structure of $C^{n+1}$. We then take $J$ to be the restriction of $J_{C^{n+1}}$ on $\xi$. We understand $D$ through the study of $\partial D$ viewed as a pseudohermitian manifold as above. On the other hand, we view the Heisenberg group $H^{n}$ as a (flat) pseudohermitian manifold $\left(R^{2 n+1}, \hat{J}, \hat{\Theta}\right)$. Here $\hat{\Theta} \equiv d z+\sum_{j=1}^{n}\left(x_{j} d y_{j}-y_{j} d x_{j}\right)$ and $\hat{J} \hat{e}_{j}=\hat{e}_{j^{\prime}}$, $\hat{J} \hat{e}_{j^{\prime}}=-\hat{e}_{j}$ where $\hat{e}_{j} \equiv \partial_{x_{j}}+y_{j} \partial_{z}, \hat{e}_{j^{\prime}} \equiv \partial_{y_{j}}-x_{j} \partial_{z} \in \operatorname{ker} \hat{\Theta}$.

Now consider a (say, $C^{\infty}$ smooth) hypersurface $\Sigma \subset(M, J, \Theta)$. There is a dichotomy for points of $\Sigma$. We call a point $p$ singular if $\xi(\equiv \operatorname{ker} \Theta)$ coincides with $T \Sigma$ at $p$. Otherwise, we call $p$ a nonsingular point where $\xi(\equiv \operatorname{ker} \Theta)$ is transverse to $T \Sigma$. At a nonsingular point in $\Sigma$, we can talk about the so called (unit) horizontal (or Legendrian) normal $\nu \in \xi$ such that $\nu$ is of unit length and perpendicular to

1991 Mathematics Subject Classification. Primary: 35L80; Secondary: 35J70, 32V20, 53A10, 49Q10.

Key words and phrases. Heisenberg group, p-minimal surface, Bernstein-type theorem. 
$\xi \cap T \Sigma$ with respect to the Levi metric $G \equiv \frac{1}{2} d \Theta(\cdot, J(\cdot))$. Consider a domain $\tilde{\Omega} \subset$ $M$ with $\Sigma=\partial \tilde{\Omega}$. By computing the first variation of the volume (natural volume element $=\Theta \wedge(d \Theta)^{n}$ which is the Euclidean volume for $H^{n}$ ) of $\tilde{\Omega}$ in the direction $\nu$, we obtain a notion of area for $\Sigma$, called $p$-area. We then define the $p$-mean curvature $H$ as the first variation of the $p$-area in the direction $\nu$ (see [1] or [4] for more details). Here we simply write down $H$ for a graph $\Sigma \equiv\left\{\left(x_{1}, y_{1}, \ldots, x_{n}, y_{n}, u\left(x_{1}\right.\right.\right.$, $\left.\left.\ldots, y_{n}\right)\right\}$ in the Heisenberg group $H^{n}$ and hence the equation of prescribed $p$-mean curvature $H$ reads

$$
\operatorname{div} \frac{\nabla u+\vec{F}}{|\nabla u+\vec{F}|}=H \text { in } \Omega \subset R^{2 n}
$$

where $u: \Omega \subset R^{2 n} \rightarrow R$ and $\vec{F} \equiv\left(-y_{1}, x_{1},-y_{2}, x_{2}, \ldots,-y_{n}, x_{n}\right)$ (also for general $\vec{F}$ ). We observed that (1.1) is a degenerate (hyperbolic and elliptic) PDE in dimension $2(n=1)$. We call a point where $\nabla u+\vec{F}=0$ in $\Omega$ singular, which is the projection of a singular point in $\Sigma$ onto $R^{2 n}$. In higher dimensions $(n \geq 2)$, we observed that (1.1) is subelliptic (square sum of $2 n-1$ vector fields satisfying Hörmander's condition) in the nonsingular domain $\{p \in \Omega \mid \nabla u+\vec{F} \neq 0$ at $p\}$. We have been working on this equation (also for general $\vec{F}$ ) in recent years $([4],[5],[6]$, [7], [2], [3], and the survey paper [1]).

For $n=1$, we can write

$$
\frac{\nabla u+\vec{F}}{|\nabla u+\vec{F}|}=(\cos \theta, \sin \theta)
$$

locally for some angle function $\theta$. By (1.2) we can write equation (1.1) (replacing $x_{1}, y_{1}$ by $\left.x, y\right)$ as follows:

$$
\operatorname{div}(\cos \theta, \sin \theta) \equiv(\cos \theta)_{x}+(\sin \theta)_{y}=H .
$$

For $u \in C^{1}(\Omega), \vec{F} \in C^{0}(\Omega)$ in a nonsingular plane domain $\Omega$, we have $\theta \in C^{0}$ locally. We may consider $\theta \in C^{0}$ as an independent variable. Define $N \equiv(\cos \theta, \sin \theta)$ such that $N$ and $N^{\perp} \equiv(\sin \theta,-\cos \theta)$ are $C^{0}$ vector fields. We also call the integral curves of $N^{\perp}$ characteristic curves.

Theorem 1.1 (Theorem A in [7]). Let $\Omega$ be a domain of $R^{2}$ and $H \in C^{0}(\Omega)$. Let $\theta \in C^{0}(\Omega)$ satisfy equation (1.3) in the weak sense, i.e.

$$
\int_{\Omega}(\cos \theta, \sin \theta) \cdot \nabla \varphi+\int_{\Omega} H \varphi=0
$$

for all $\varphi \in C_{0}^{\infty}(\Omega)$. Let $\Gamma \subset \Omega$ be a ( $C^{1}$ smooth) characteristic curve with $\sigma$ being the unit-speed parameter. Then $\Gamma$ is $C^{2}$ smooth and the curvature of $\Gamma$ (along $N^{\perp}$ direction) equals $-H$, that is, $\frac{d \theta}{d \sigma}=-H$.

We remark that in Theorem A of [13], Pauls proved the case of $H=0$ in which $\Gamma$ is a straight line under the extra condition that components of the horizontal Gauss map (i.e., $\frac{\nabla u+\vec{F}}{|\nabla u+\vec{F}|}$ in our notation with $\vec{F}=(-y, x)$ ) are $W^{1,1}(\Omega)$. In Theorem 1.1 above, if $\theta$ satisfies (1.4), we proved that $\Gamma$ is a minimizer for the following energy functional:

$$
L_{H}(\Gamma) \equiv|\Gamma|-\int_{\Omega_{\Gamma}} H d x d y
$$


where $|\Gamma|$ denotes the length of $\Gamma$ and $\Omega_{\Gamma}$ is the domain surrounded by $\Gamma$ and some line segments (see Section 2 in [7]). So the basic calculus of variation tells us that the curvature of $\Gamma$ (along $N^{\perp}$ direction) equals $-H$ without invoking extra regularity assumption. Also $H$ is only required to be $C^{0}$. In [11] Monti and Rickly considered the case of $H=$ constant for a convex isoperimetric set. We do not need convexity in Theorem 1.1.

\section{UNIQUENESS OF CHARACTERISTIC AND SEED CURVES}

For $\theta \in C^{0}, N\left(N^{\perp}\right.$, resp. $) \equiv(\cos \theta, \sin \theta)(\equiv(\sin \theta,-\cos \theta)$, resp. $)$ is a $C^{0}$ vector field. Then for any $p \in \Omega$, there exists at least one integral curve of $N$ $\left(N^{\perp}\right.$, resp.), called seed curve (characteristic curve, resp.) passing through $p$. The uniqueness of integral curves for a $C^{0}$ vector field does not hold true in general (see page 18 in [9]). Let $p \in \Omega$ and $B_{r}(p) \equiv\{q \in \Omega|| q-p \mid<r\}$. Define $H_{M}(r) \equiv$ $\max _{q \in \partial B_{r}(p)}|H(q)|$.

Theorem 2.1 (Theorem $B$ in [7]). (a) Let $\theta \in C^{0}(\Omega)$ and $H \in L_{\text {loc }}^{1}(\Omega)$ satisfy (1.4). Let $p \in \Omega$ and suppose there is $r_{0}>0$ such that $B_{r_{0}}(p) \subset \subset \Omega$ and

$$
\int_{0}^{r_{0}} H_{M}(r) d r<\infty .
$$

Then there is $r_{1}, 0<r_{1} \leq r_{0}$, such that there exists a unique seed curve passing through $p$ in $B_{r_{1}}(p)$.

(b) Let $\theta \in C^{0}(\Omega)$ and $H \in C^{0,1}(\Omega)$ (Lipschitzian) satisfy (1.4). Then for any point $p \in \Omega$, we can find $r_{1}>0$ such that there exists a unique characteristic curve passing through $p$ in $B_{r_{1}}(p)$.

In Theorem 2.1 (b), if $H$ is only continuous, we gave an example for the nonuniqueness of characteristic curves (see Example 3.2 in [7]). Note that $u$ is not involved in Theorems 1.1 and 2.1. Now we consider $u$. Let $u \in C^{1}$ and $\vec{F}=\left(F_{1}, F_{2}\right) \in C^{1}$. Recall that a point $p \in \Omega \subset R^{2}$ is called singular (nonsingular, resp.) if $\nabla u+\vec{F}=$ $0(\neq 0$, resp. $)$ at $p$. At a nonsingular point, we set $N \equiv N^{u} \equiv \frac{\nabla u+\vec{F}}{|\nabla u+\vec{F}|}$. We call $\Omega$ nonsingular if every point of $\Omega$ is not singular. We had another uniqueness theorem for characteristic curves.

Theorem 2.2 (Theorem $B^{\prime}$ in [7]). Let $u: \Omega \subset R^{2} \rightarrow R$ be a $C^{1}$ smooth function such that $\Omega$ is a nonsingular domain with $\vec{F} \in C^{1}(\Omega)$. Then for any point $p \in \Omega$, we can find $r_{1}>0$ such that $B_{r_{1}}(p) \subset \Omega$ and there exists a unique characteristic curve passing through $p$ in $B_{r_{1}}(p)$.

In Theorem 2.2, we only assume $u, \vec{F} \in C^{1}$, and do not use any property of $H$, which is different from Theorem 2.1 (b). Even for the case $H=0$, seed curves may only be $C^{1}$ smooth, but not $C^{2}$ smooth (see the remark after the proof of Theorem D in Section 5 of [7]). However if $N \equiv(\cos \theta, \sin \theta)$ arises from $u$ (i.e., $N=N^{u}$ ), Pauls ([13]) proved that when $u \in C^{1}, \theta \in C^{0} \cap W^{1,1}$, and $H=0$, then the seed curves are $C^{2}$ smooth. In Theorem 3.4 below, we proved the same conclusion under the condition that $u \in C^{1}\left(\theta \in C^{0}\right.$ follows) and $H \in C^{1}$ (in fact, that $H \in C^{0}$ and only $C^{1}$ in the $N$ direction is enough). 


\section{Regularity of $\theta$ AND Characteristic AND SEED CURVES}

Suppose that $\theta$ arises from $u$, i.e., $(\cos \theta, \sin \theta)=N^{u}$. Let $S(u)$ denote the set of singular points. Suppose that $u \in C^{1}(\Omega)$ satisfies

$$
\int_{\Omega} N^{u} \cdot \nabla \varphi+\int_{\Omega} H \varphi=0
$$

for all $\varphi \in C_{0}^{\infty}(\Omega)$ with $\vec{F} \in C^{1}(\Omega)$ and $S(u)$ being empty in $\Omega$. Can we have higher order regularity for $u$, say, $u \in C^{2}$ ? This is impossible as shown by the following example. Let $u_{g} \equiv x y+g(y)$ where $g \in C^{1} \backslash C^{2}$. Then $u_{g}$ satisfies (3.1) with $H$ $=0, \vec{F}=(-y, x)$ on any nonsingular domain $\Omega$ for $u_{g}$. On the other hand, the characteristic and seed curves associated to $u_{g}$ are all the same for different $g$ 's. That is, the differentiability of $g$ determines the differentiability of $u_{g}$, but does not affect the shape of characteristic and seed curves. On the other hand, we proved that $\theta$ is in fact $C^{1}$ smooth (hence $N \in C^{1}$, but not $u \in C^{2}$ ) (see Theorem 3.4 below). Before doing this we need to introduce some kind of special coordinates. These coordinates play an important role in understanding the local structure.

Definition 3.1. Let $N$ be a $C^{0}$ vector field with $|N| \equiv 1$ on a domain $\Omega \subset R^{2}$. A system of $C^{1}$ smooth local coordinates $s, t$ is called a system of characteristic coordinates if $s$ and $t$ have the property that $\nabla s \| N^{\perp}$ and $\nabla t \| N$, i.e., $\nabla s$ and $\nabla t$ are parallel to $N^{\perp}$ and $N$, resp.. It follows that $\{t=$ constants $\}$ are characteristic curves while $\{s=$ constants $\}$ are seed curves.

Let $D \equiv|\nabla u+\vec{F}|$. For a plane $C^{1}$ vector field $\vec{F}=\left(F_{1}, F_{2}\right)$, we define

$$
\operatorname{rot} \vec{F} \equiv\left(F_{2}\right)_{x}-\left(F_{1}\right)_{y} \text {. }
$$

Theorem 3.2 (existence of characteristic coordinates [7]). Let $u: \Omega \subset R^{2} \rightarrow$ $R$ be a $C^{1}$ smooth solution to (3.1) ( $\Omega$ being nonsingular) with $\vec{F} \in C^{1}(\Omega)$ and $H \in C^{0}(\Omega)$. Then for any point $p_{0} \in \Omega$ there exist a neighborhood $\Omega^{\prime} \subset \Omega$ and real functions $s, t \in C^{1}\left(\Omega^{\prime}\right)$ such that $\{t=$ constants $\}$ and $\{s=$ constants $\}$ are characteristic curves and seed curves, respectively. Moreover, there are positive functions $f, g \in C^{0}\left(\Omega^{\prime}\right)$ such that

$$
\nabla s=f N^{\perp}, \nabla t=g D N
$$

Also $N f$ and $N^{\perp} g$ exist and are continuous in $\Omega^{\prime}$. In fact, $f$ and $g$ satisfy the following equations

$$
N f+f H=0, N^{\perp} g+\frac{(\operatorname{rot} \vec{F}) g}{D}=0 .
$$

For a perhaps smaller neighborhood $\Omega^{\prime \prime} \subset \Omega^{\prime}$ of $p_{0}$, the map $\Psi:(x, y) \in \Omega^{\prime \prime} \rightarrow$ $(s, t) \in \Psi\left(\Omega^{\prime \prime}\right)$ is a $C^{1}$ diffeomorphism such that

$$
\Psi^{*}\left(\frac{d s^{2}}{f^{2}}+\frac{d t^{2}}{g^{2} D^{2}}\right)=d x^{2}+d y^{2}
$$

We remark that the existence of $C^{1}$ smooth $s$ can be proved for $N$ satisfying (1.4) (i.e., not defined by $u$ ) instead of (3.1) (see Theorem 4.1 in [7]). 
Corollary 3.3 (Corollary C.1 in [7]). Suppose we are in the situation of Theorem 3.2. Then $\theta$ is $C^{1}$ smooth in $s$ and there holds

$$
\theta_{s} \equiv \frac{\partial \theta}{\partial s}=-\frac{H}{f} \text {. }
$$

Since $f$ is $C^{1}$ smooth in $t, \theta_{s}$ is also $C^{1}$ smooth in $t$ if we assume that $H$ has the same property according to (3.2). In fact, we proved that $\theta$ is $C^{1}$ smooth in $t$ too, and hence $\theta \in C^{1}$. That is, $\theta$ gains one derivative.

Theorem 3.4 (regularity of $\theta[7]$ ). Let $u: \Omega \subset R^{2} \rightarrow R$ be a $C^{1}$ smooth (weak) solution to $\operatorname{div} N^{u}=H$ in $\Omega$ ( $\Omega$ being nonsingular) with $\vec{F} \in C^{1}(\Omega)$ and $H \in$ $C^{0}(\Omega)$. Suppose $N(H)$ exists and is continuous. Then $\theta \in C^{1}$ and the characteristic and seed curves are $C^{2}$ smooth. Moreover, $N^{\perp} D$ exists and is continuous in $\Omega$. In $(s, t)$ coordinates near a given point as in Theorem 3.2, we have

$$
\theta_{t} \equiv \frac{\partial \theta}{\partial t}=\frac{r o t \vec{F}}{g D^{2}}-\frac{N^{\perp}(\log D)}{g D}=\frac{1}{g D^{2}}\left(\operatorname{rot} \vec{F}-N^{\perp} D\right) .
$$

In case $H=0$ or a constant, we can prove Theorem 3.4 directly from the precise parametric expression of $x$ or $y$. The situation $H=$ a nonzero constant arises from considering the boundary of an isoperimetric set in the 3-dimensional Heisenberg group $H^{1}$. It has been conjectured (e.g., [12]) that an isoperimetric set in $H^{1}$ is congruent with a certain type of sphere. Based on the analysis of the singular set and characteristic curves ([4]) for a $C^{2}$ smooth surface, Ritoré and Rosales ([14]) proved such a conjecture for isoperimetric sets of class $C^{2}$ without any symmetry assumption. Later Monti and Rickly ([11]) obtained the same result for convex isoperimetric sets without regularity assumption.

According to (3.2), we have good control for $\theta$ along the characteristic curves, i.e. the $s$-direction. If the control for $\theta$ fails along a seed curve, i.e. $t$-direction, say, at some $s_{0}$, then we showed that it fails also for $s$ near $s_{0}$. That is, the jump of a certain concerned quantity is kept in short "s-time" along the characteristic curves. This ends up to reach a contradiction. We borrowed the idea of conveying information along the characteristic curves from the study of hyperbolic P.D.E. (e.g., $[8])$.

\section{Regularity of the $p$-AREA Element AND THE Singular SET}

Recall that $D d x \wedge d y \equiv|\nabla u+\vec{F}| d x \wedge d y$ is the $p($ or $H)$-area element for a graph defined by $u$. For $u \in C^{1}$, we have $D \in C^{0}$. Suppose that $u \in C^{1}(\Omega)$ satisfies (3.1) with $\vec{F} \in C^{1}(\Omega)$ and $H \in C^{1}(\Omega)$, say ( $\Omega$ being nonsingular). It follows from Theorem 3.4 that $N^{\perp} D$ exists and is continuous in $\Omega$. Observe that $\frac{\partial}{\partial t}=\frac{1}{g D} N$ by Theorem 3.2. It follows from (3.2) and Theorem 3.2 that $\left(\theta_{s}\right)_{t}$ exists and is continuous. Then by a basic fact in calculus (see Lemma 5.4 in [7]) we conclude that $\left(\theta_{t}\right)_{s}$ exists and equals $\left(\theta_{s}\right)_{t}$ (in particular, $N^{\perp} N^{\perp} D$ exists and is continuous). Computing from (3.2) and (3.3), we also obtain an O.D.E. of second order for $D$ (see below). Denote $N^{\perp} D$ and $N^{\perp} N^{\perp} D$ by $D^{\prime}$ and $D^{\prime \prime}$, resp.. 
Theorem 4.1 (regularity of $D$ and equation for $D[5]$ ). Suppose we are in the situation of Theorem 3.4. Then $D^{\prime \prime}$ exists and is continuous in $\Omega$. Moreover, $D$ satisfies the following equation:

$$
\begin{aligned}
D D^{\prime \prime}= & 2\left(D^{\prime}-\frac{\operatorname{rot} \vec{F}}{2}\right)\left(D^{\prime}-\operatorname{rot} \vec{F}\right) \\
& +\left(N^{\perp} \operatorname{rot} \vec{F}\right) D+\left(N H+H^{2}\right) D^{2} .
\end{aligned}
$$

For $\vec{F}=(-y, x)$ (p-mean curvature case), we have $\operatorname{rot} \vec{F}=2$. In this case, (4.1) is reduced to

$$
D D^{\prime \prime}=2\left(D^{\prime}-1\right)\left(D^{\prime}-2\right)+\left(N H+H^{2}\right) D^{2} .
$$

If, furthermore, $H=0$ in (4.2), the equation $D D^{\prime \prime}=2\left(D^{\prime}-1\right)\left(D^{\prime}-2\right)$ is integrable. Namely, we observed that

$$
\begin{aligned}
\frac{2 D^{\prime}}{D} & =\frac{D^{\prime} D^{\prime \prime}}{\left(D^{\prime}-1\right)\left(D^{\prime}-2\right)} \\
& =\frac{-D^{\prime \prime}}{D^{\prime}-1}+\frac{2 D^{\prime \prime}}{D^{\prime}-2} \\
& =-\frac{\left(D^{\prime}-1\right)^{\prime}}{D^{\prime}-1}+\frac{2\left(D^{\prime}-2\right)^{\prime}}{D^{\prime}-2}
\end{aligned}
$$

at points of a characteristic curve (line), where $D^{\prime} \neq 1,2$. Integrating (4.3), we obtained

$$
\left|D^{\prime}-2\right|^{2}=c\left|D^{\prime}-1\right| D^{2}
$$

for some constant $c>0$. It is not hard to see that when a nonsingular point tends to a singular point along a characteristic curve (line), either $D^{\prime}$ goes to 2 or $D^{\prime}$ goes to 1 ( $D^{\prime} \equiv 1$ in fact in this case). More generally, we had the following result.

Theorem 4.2 (behavior of $D^{\prime}$ near a singular point [5]). Suppose we are in the situation of Theorem 3.4. Suppose $\mathrm{NH}+\mathrm{H}^{2}$ is bounded on a characteristic curve $\Gamma$ approaching a singular point $p$. Then either $D^{\prime}$ converges to 1 or $D^{\prime}$ converges to 2 along $\Gamma$ as points tend to $p$ (where $D=0$ ).

Applying equation (4.2) and Theorem 4.2 to analyze the singular set of a $C^{1}$ smooth solution to (3.1) with $\vec{F}=(-y, x)$ is one of strategies in an on-going project. For instance, we can show the impossibility of connecting two distinct singular points (where $D=0$ ) by a characteristic curve under a mild condition on $H$. For if possible, there exists a point on this characteristic curve with maximum $D$-value, and hence $D^{\prime}=0$. Evaluating equation (4.2) at this point gives

$$
0 \geq D^{\prime \prime}=\frac{4}{D}+\left(N H+H^{2}\right) D>0
$$

if $N H \geq 0$ (including the $H=$ constant case), a contradiction. 


\section{REFERENCES}

[1] Cheng, J.-H., Surfaces of prescribed p-mean curvature in the Heisenberg group, Proceedings of the 4th ICCM, Dec.17-22, 2007, Hangzhou, China, Vol II, 40-58.

[2] Cheng, J.-H. and Hwang, J.-F., Properly embedded and immersed minimal surfaces in the Heisenberg group, Bull. Aus. Math. Soc., 70 (2004) 507-520.

[3] Cheng, J.-H. and Hwang, J.-F., Variations of generalized area functionals and P-area minimizers of bounded variation in the Heisenberg group, preprint.

[4] Cheng, J.-H., Hwang, J.-F., Malchiodi, A., and Yang, P., Minimal surfaces in pseudohermitian geometry, Scuola Norm. Sup. Pisa Cl. Sci. (5), 4 (2005) 129-177.

[5] Cheng, J.-H., Hwang, J.-F., Malchiodi, A., and Yang, P., The singular set of $C^{1}$ smooth surfaces with prescribed p-mean curvature in the Heisenberg group, in preparation.

[6] Cheng, J.-H., Hwang, J.-F., and Yang, P., Existence and uniqueness for p-area minimizers in the Heisenberg group, Math. Ann., 337 (2007) 253-293.

[7] Cheng, J.-H., Hwang, J.-F., and Yang, P., Regularity of $C^{1}$ smooth surfaces with prescribed p-mean curvature in the Heisenberg group, arXiv: 0709.1776v1 [math.DG] 12 Sep 2007.

[8] Evans, L. C., Partial Differential Equations, Graduate Studies in Mathematics, Vol. 19, Amer. Math. Soc., 1998.

[9] Hartman, P., Ordinary Differential Equations, 2nd ed., SIAM edition, 2002.

[10] Lee, J. M., The Fefferman metric and pseudohermitian invariants, Trans. Amer. Math. Soc., 296 (1986) 411-429.

[11] Monti, R. and Rickly, M., Convex isoperimetric sets in the Heisenberg group, arXiv: math.DG/0607666 v1.

[12] Pansu, P., Une inégalité isopérimétrique sur le groupe de Heisenberg, C.R.Acad.Sci.Paris Sér. I Math. 295 (1982) 127-130.

[13] Pauls, S. D., H-minimal graphs of low regularity in $H^{1}$, Comment. Math. Helv. 81 (2006) 337-381; arXiv: math.DG/0505287 v3, Nov. 1, 2006 (to which the reader is referred).

[14] Ritoré, M. and Rosales, C., Area-stationary surfaces in the Heisenberg group $H^{1}$, preprint.

Institute of Mathematics, Academia Sinica, Taipei, Taiwan, R.O.C.

E-mail address: cheng@math.sinica.edu.tw 